Kleinbach, Karlheinz; Franz, Ulrich

\title{
Von der Analyse der Schwachstellen zu einem neuen Orientierungskonzept
}

formal überarbeitete Version der Originalveröffentlichung in:

formally revised edition of the original source in:

Verkehr und Technik (2008) 12, S. 469-473

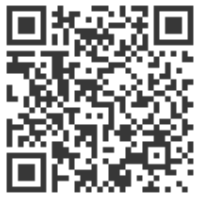

Bitte verwenden Sie in der Quellenangabe folgende URN oder DOI /

Please use the following URN or DOI for reference:

urn:nbn:de:0111-pedocs-116020

10.25656/01:11602

https://nbn-resolving.org/urn:nbn:de:0111-pedocs-116020

https://doi.org/10.25656/01:11602

\section{Nutzungsbedingungen}

Gewährt wird ein nicht exklusives, nicht übertragbares, persönliches und beschränktes Recht auf Nutzung dieses Dokuments. Dieses Dokument ist ausschließlich für den persönlichen, nicht-kommerziellen Gebrauch bestimmt. Die Nutzung stellt keine Übertragung des Eigentumsrechts an diesem Dokument dar und gilt vorbehaltlich der folgenden Einschränkungen: Auf sämtlichen Kopien dieses Dokuments müssen alle Urheberrechtshinweise und sonstigen Hinweise auf gesetzlichen Schutz beibehalten werden. Sie dürfen dieses Dokument nicht in irgendeiner Weise abändern, noch dürfen Sie dieses Dokument für öffentliche oder kommerzielle Zwecke vervielfältigen, öffentlich ausstellen, aufführen, vertreiben oder anderweitig nutzen.

Mit der Verwendung dieses Dokuments erkennen Sie die Nutzungsbedingungen an.

\section{Terms of use}

We grant a non-exclusive, non-transferable, individual and limited right to using this document.

This document is solely intended for your personal, non-commercial use. Use of this document does not include any transfer of property rights and it is conditional to the following limitations: All of the copies of this documents must retain all copyright information and other information regarding legal protection. You are not allowed to alter this document in any way, to copy it for public or commercial purposes, to exhibit the document in public, to perform, distribute or otherwise use the document in public.

By using this particular document, you accept the above-stated conditions of use.

\section{Kontakt / Contact:}

peDOcs

DIPF | Leibniz-Institut für Bildungsforschung und Bildungsinformation Informationszentrum (IZ) Bildung

E-Mail: pedocs@dipf.de

Internet: www.pedocs.de 
$\begin{array}{lr}\text { Abstract } & 2 \\ \text { Einleitung } & 3 \\ \text { Von der Analyse der Schwachstellen zu einem neuen Orientierungskonzept } & 3 \\ \text { Die Berücksichtigung kognitiver Karten im Orientierungssystem } & 6 \\ \text { Wie lassen sich individuelle Konzepte mit vorhandenen komplexen Netzplänen } & 6 \\ \text { und Karten verbinden? } & 7 \\ \text { Individuelle Anforderungen an ein Informationssystem zeichnen sich aus durch } \\ \text { einen niedrigen Komplexitätsgrad } & 7 \\ \text { Inklusive Anforderungen an ein Informationssystem } & 13 \\ \text { Evaluation } & 15 \\ \text { Barrierefreiheit für Menschen mit geistiger Behinderung als gemeinsame } & 15 \\ \text { Aufgabe } & 17 \\ \text { Literatur } & \\ \text { Grafiken: } & \end{array}$

Dr. Karlheinz Kleinbach und Ulrich Franz

Fakultät für Sonderpädagogik Reutlingen

Institut für Allgemeine Sonderpädagogik

Pestalozzistr. 53

72762 Reutlingen

07121-271-9342

kleinbach@ph-ludwigsburg.de

Zuerst in: Verkehr und Technik 12/2008, 469 - 473;

http://www.VTdigital.de/VT.12.2008.469 


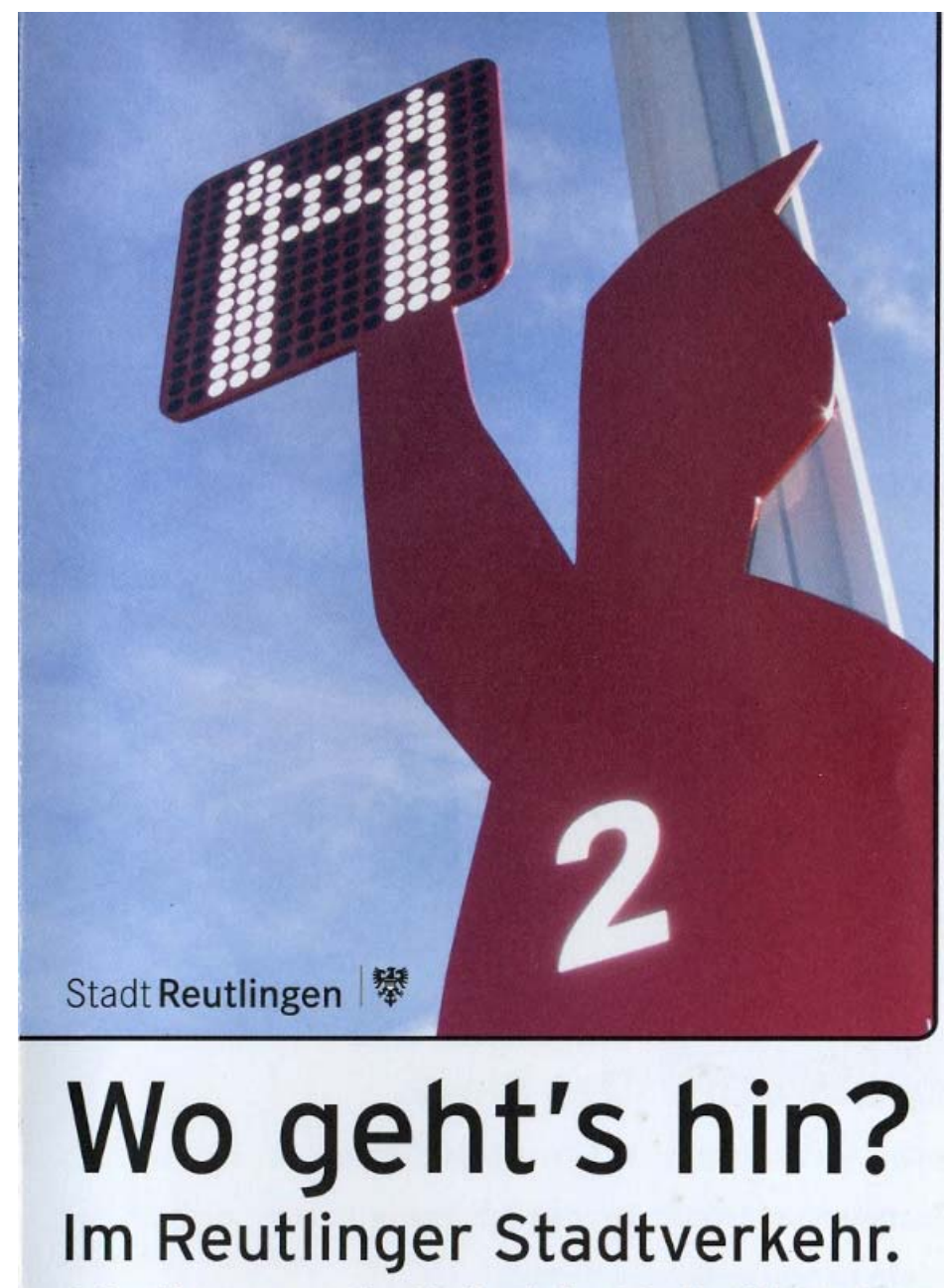

Orientierung an der Haltestelle Stadtmitte (ZOB)

\section{Abstract}

In einer Zeit des Wandels vom bürokratischen Sozialstaat in einen sozialen Bürgerstaat sind für Menschen mit geistiger Behinderung gesellschaftliche Teilhabe und ein möglichst hohes Maß an Selbstbestimmung zu gewährleisten. Deshalb gewinnt Mobilität die Bedeutung einer Leitkompetenz für die selbständige Lebensführung. Planung, Implementierung und Evaluation des Reutlinger Orientierungssystem zur Mobilitätserweiterung werden vorgestellt. Absicht und Ziel dieses System ist es, die Nutzung des Busangebots der Reutlinger Stadtverkehre für alle Menschen zu erleichtern. In diesem inklusiven Anspruch liegt die besondere Bedeutung des Reutlinger Konzeptes. Neu und bisher einzigartig ist dabei, dass in diesem Vorhaben Verkehrsplaner der Stadt Reutlingen, ein Grafiker sowie Busunternehmer gemeinsam mit Menschen mit Behinderung ein Orientierungs- und Leitsystem entwickelt haben. Umgesetzt und sichtbar ist dies an der Neugestaltung der Haltestellen, der Fahrpläne, den Markierungen und Linienbezeichnungen in und an den Bussen und am Zentralen Omnibusbahnhof der Stadt. Menschen mit Lernbeeinträchtigungen wurden dabei als Experten für Mobilitätsbarrieren zu Kooperationspartnern in Planung und Gestaltung. Barrierefreiheit beginnt in unseren Köpfen in dem wir Hürden, Sperren und Erschwernisse als gemeinsame soziale und 
städtebauliche Aufgabe verstehen und dies bei konkreten Vorhaben auch in den politischen Gremien vertreten und uns an der Realisierung verantwortlich beteiligen.

\section{Einleitung}

Der Wandel unserer Lebenswelt, die Veränderung rechtlicher und gesellschaftlicher Rahmenbedingungen sowie die technischen Möglichkeiten eröffnen Menschen mit geistiger Behinderung neue Möglichkeiten eines selbstbestimmten Lebens. Wohnen, Arbeits- und Beschäftigungsmarkt, Zugänge zu kulturellen Angeboten und das Grundrecht auf chancengleiche Bildung und Erziehung sind dabei in mancherlei Weise abhängig selbständigen Nutzung des öffentlichen Personennahverkehrs. Dies stellt in pädagogischer Hinsicht eine besondere Herausforderung dar. Denn der größte Teil der Kinder und Jugendlichen mit Lernbeeinträchtigungen wird noch immer mit speziell eingerichteten Fahrdiensten zum Unterricht in die Schule gefahren und wieder nach Hause gebracht. Vor zwei Jahren ließ das bayrische Kultusministerium an den Entlass-Stufen der Schulen für Geistigbehinderte eine Befragung dazu durchführen. Im Abschlussbericht dieser Befragung ist zu lesen: „Die Tatsache, dass nur 101 Schüler von immerhin 3076 Schülern (das sind 3,2\%) dieser Stufe ihren Schulweg selbständig bewältigen, scheint auf geringe Selbständigkeit hinzuweisen“ (STAATSINSTITUT 2006, 7).

Solche Immobilitätsfallen betreffen allerdings keineswegs nur Kinder und Jugendlichen mit Lernbeeinträchtigungen. Es liegen zwischenzeitlich eine Reihe von Untersuchungen vor, die nachweisen wie das elterliche Verhalten trägt dazu beiträgt, dass Kinder immer weniger selbständig am Verkehr teilnehmen (KOHLER 2002, TULLY/SCHULZ 1999, HERRY/SAMMER 1998). Es entsteht die paradoxe Situation, in der Kinder sogar zu ihren Freizeitorten mit dem Pkw gebracht werden, „um vor den Gefahren des hohen Verkehrsaufkommens geschützt zu werden. Obwohl die Kinder in der Befragung unmissverständlich ihre Kritik am Autoverkehr zum Ausdruck brachten, so stellen sie gleichzeitig - wenn auch unfreiwillig - dessen eifrigste Nutzer dar" (KOHLER 2002, 114).

Durch Beschluss eines Antrags der Frauenliste ließ der Reutlinger Gemeinderat im Jahre 1999 prüfen, wie sich die Orientierung von Menschen mit Behinderung im öffentlichen Personennahverkehr (ÖPNV) verbessern lässt („Brauchen Menschen mit Behinderung ein spezifisches Leitsystem um sich bei der Fahrt mit dem Stadtbus zu orientieren?"). Dabei verstanden Stadtverwaltung und Stadtwerke Reutlingen als Träger des ÖPNV diese Anfrage der Frauenliste nicht nur als eine an sie gerichtete und von ihnen zu lösende Aufgabe auf. Vielmehr sollten Nutzerinnen und Nutzer in die Planung und Realisierung des bedarfsorientierten Konzepts einbezogen werden. Dies führte 2002 zu einer Kooperation des Reutlinger Arbeitskreises Selbstbestimmung mit der Stadtverwaltung, den Verkehrsunternehmen, dem Grafiker Ulrich Franz sowie Studierenden der Fakultät für Sonderpädagogik der Pädagogischen Hochschule Ludwigsburg in Reutlingen.

\section{Von der Analyse der Schwachstellen zu einem neuen Orientierungskonzept}

Welche Schwierigkeiten haben Menschen mit Einschränkungen der Wahrnehmung, mit Orientierungsproblemen, ohne ausreichende Orts- und Sprachkenntnisse bei der Nutzung der Reutlinger Stadtbusse? Wodurch sind solche Schwierigkeiten verursacht und wodurch werden diese möglicherweise verstärkt? Mit welchen 
individuellen und/oder systembezogenen Erleichterungen können Nutzerinnen und Nutzer unterstützt werden? Erweitert sich dadurch ihre Mobilität? Durch Beobachtung, Befragung und Analyse konnten unterschiedliche Nutzungsprofile ermittelt werden (Kasten 1), eine Mängellisten erstellt, Änderungsvorschläge gesammelt und klassifiziert werden, und schließlich eine Übersicht einzelner Aufmerksamkeiten und Fragestellungen erarbeitet werden.

- Fehlende Koordinierung der Anzeigen, räumlich und inhaltlich.

- Unterschiedliche Ebenen sind vermischt: Inhalt, Weg, Daten

- Informationen aus Plänen sind schlecht in der Wirklichkeit wieder zu finden.

- Inhalten sind visuell schwer erkennbar, grafisch und typografisch.

- Verwendung unterschiedlicher grafischer Systeme (z.B. unterschiedlich umgesetzte Kartenansichten)

Kasten 1: Mängelliste

Es zeigte sich dabei, dass für behinderte und benachteiligte Menschen die Orientierung im ÖPNV individuell in ganz unterschiedlicher Weise prekär und labil ist. Für Menschen mit eingeschränkten motorischen und kognitiven Möglichkeiten verbindet sich Wirklichkeit nicht immer und selbstverständlich mit Wirksamkeit: Wahrnehmung und Vergewisserung über den aktuellen Aufenthaltsort, ebenso wie Möglichkeiten der Verständigung sind möglicherweise sehr unterschiedlich. An welche technischen, organisatorischen und betriebswirtschaftlichen Anforderungen muss sich der Anbieter orientieren? Wie lassen sich diese individuellen Voraussetzungen und Bedarfe mit dem allgemeinen System des Verkehrsverbundes angemessen vermitteln? Lässt sich eine Kooperationsebene formulieren und theoretisch beschreiben, die produktiver ist als die Verhandlung über Beseitigung problematischer Details? Die in Kasten 2 dargestellte Übersicht versucht diese beiden Anforderungen (,front-end' und ,back-end') zu vermitteln. 


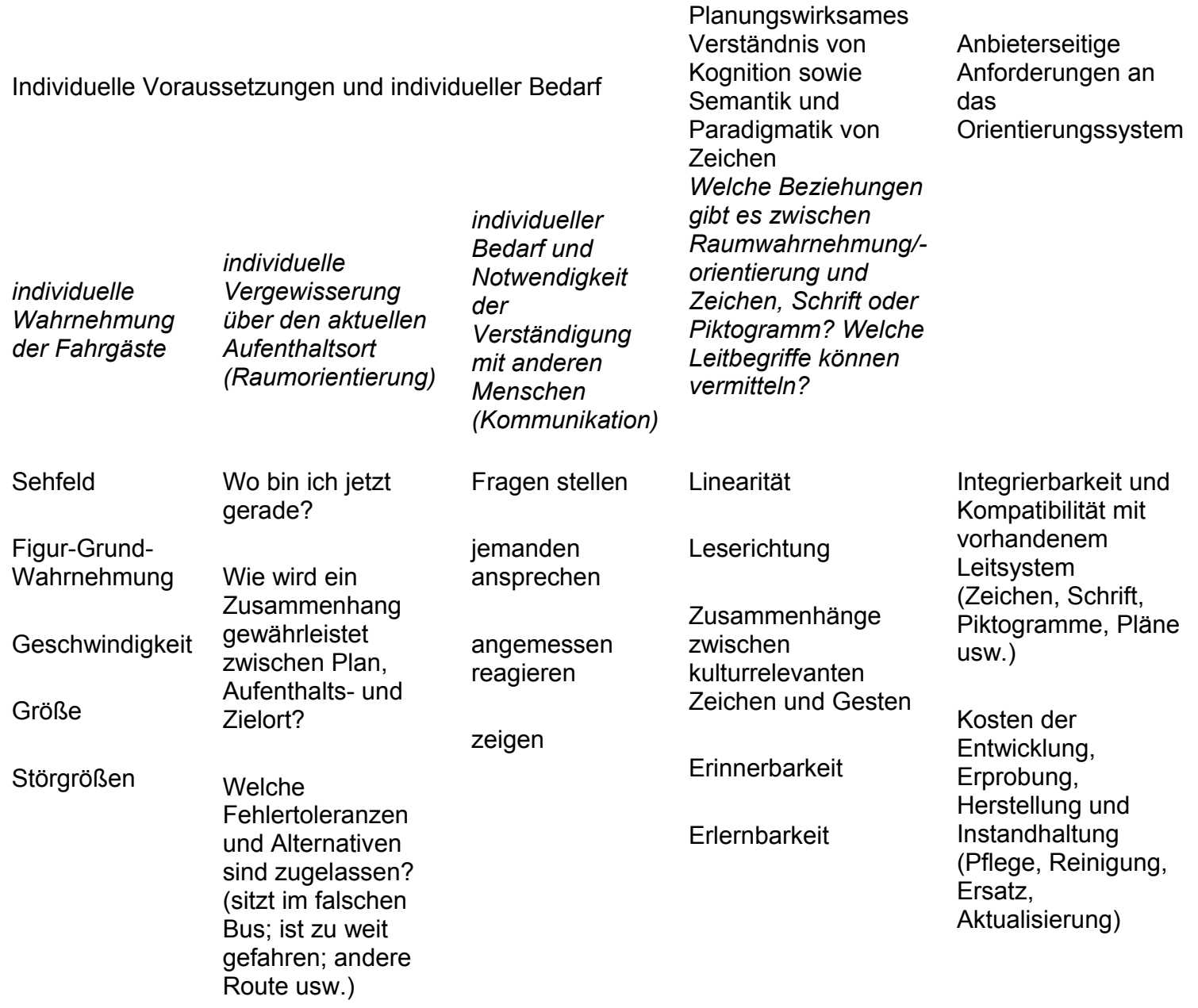

Kasten 2: individuelle und systematische Voraussetzungen für die Gestaltung eines

\section{Orientierungssystems}

In dieser Kooperation trafen unterschiedliche Wahrnehmungen aufeinander. In zahlreichen Gesprächen konnten die Mitglieder des Arbeitskreises deutlich machen, dass „gut gemeint“ für die Betroffenen nicht auch „sinnvoll und nützlich“ bedeutet. Ebenso musste der Arbeitskreis allerdings auch erfahren, wie komplex die Teilbereiche des Systems ÖPNV miteinander vernetzt und voneinander abhängig sind. Es ging dabei um die Gestaltung technischer und organisatorischer Strukturen und Vorgaben für graphische Leitsysteme, die für die Nutzer des ÖPNV üblicherweise unter der Benutzeroberfläche bleiben. Daraus wurde das gemeinsame Vorhaben „Mobilitätserweiterung durch ein Orientierungssystem“. Dieses wurde im Jahr 2002 ins 2. Innovationsprogramm ÖPNV Baden-Württemberg aufgenommen und mit 50\% Landesmitteln bezuschusst. Finanziert wurde das Projekt von der Stadt Reutlingen mit insgesamt 50 Tsd. Euro.

In der Zusammenarbeit wurde bald klar worin das Expertentum der Arbeitsgruppe Selbstbestimmung besteht. Deren Augenmerk lag gar nicht so sehr nur auf der 
Sammlung offensichtlicher Systemmängel ${ }^{1}$. Vielmehr wurde deutlich, dass Nutzungsanlässe als ,Sätze' formuliert werden. Was ist damit gemeint?

\section{Die Berücksichtigung kognitiver Karten im Orientierungssystem}

Wer den Bus nutzt fährt zur Arbeit, zur Schule zum Einkaufen, trifft Bekannte, macht einen Besuch. Die üblichen Karten und tabellenorientierten Konzepte und Leitsysteme unterscheiden sich von solchen individuellen ,Sätzen' (\#verweis auf welche Tabelle?). Was mit ,Sätzen' gemeint ist lässt sich lässt sich aus Forschungsansätzen zur kognitiver Kartierung und subjektiver Stadtgeografie gewinnen (BIRKENHAUER 2004, CASTNER 1995, DOWNS/STEA 1985, HARD 2003, HASSE 2007, JANZEN/HAWLIK 2005, MALLOT 2006).

Die Fragen dieser Forschungsansätze von Stadtgeografie und Raumpsychologie beziehen sich auf Wegorientierung. Wie finden sich Menschen in urbanen Landschaften zurecht? Mit welchen Strategien orientieren sie sich in alltäglicher und fremder Umgebung? Welche Landmarks sind für sie dabei bedeutungsrelevant? Die ,Kognitive Karte' hat sich dabei als ertragreiches theoretisches Konstrukt erwiesen. Diese ist nicht identisch ist mit ,Landkarte' oder ,Netzkarte' (BITTER 1999, 94, KLIPPEL 2002, 62; FREITAG 1997,136, MONTELLEO 1998, 93). Landkarte zeichnen sich aus durch Maßstab, Zweidimensionalität, Indexikalität und einen Zeichenschlüssel. Dagegen sind kognitive Karten raumbezogene Gedächtnis- und Orientierungsstrukturen (BITTER 1999, 94). Auch sie stellen räumliche Wirklichkeit dar, jedoch individuell, maßungebunden, multisensorisch und häufig mehrdimensional, Über die Analyse von Zeichnungen und verbalen Wegbeschreibungen lässt sich Aufschluss über Qualität und Struktur solcher kognitiven Karten gewinnen ((BITTER1999, 95f; HÜTTENMOSER 2005). Wie sich in kognitiven Karten Raumvorstellungen repräsentieren ist in der Fachliteratur bisher nur in Ansätzen erforscht (GRAMANN et al 2006; JANSEN-OSMANN 2007; JANZEN 2005; MALLOT 2006; SCHUHMANN-HENGSTLER 2006). Gesichert ist, dass der Erwerb von Raumwissen mit der Eigenbewegung im Raum zusammenhängt, sich ebenso auch aus graphischen und verbalen Referenzen aufbauen kann. Die oben genannte Forschungsansätze zeigen jedoch übereinstimmend eine Entwicklungsrichtung der Orientierung vom Knotenpunktwissen (landmark knowledge) über Streckenwissen (route knowledge) zum Überblickswissen (survey knowledge). Mit Streckenwissen ist die zielführende Abfolge von (subjektiven) Landmarks und deren Darstellung durch Sätze gemeint, die für alltägliche Wege ausreichend sind. Solche zielführenden Abfolgen kommunizieren wir - mehr oder weniger erfolgreich - wenn uns jemand nach dem Weg fragt.

\section{Wie lassen sich individuelle Konzepte mit vorhandenen komplexen Netzplänen und Karten verbinden?}

Im Rahmen eines angebotsorientierten Zugangs, also der Verbesserung eines vorhandenen Systems, sollten deshalb jene individuell bedeutsame Elemente berücksichtigt werden, diese müssen jedoch konventionell sein, d.h. allgemein verständlich und anschlussfähig bestehende Orientierungssysteme und deshalb

${ }^{1}$ hierzu gehören u.a. die grafische Gestaltung der ausgehängten Fahrpläne, die die Orientierung im Plan und das Auffinden, Vergleichen von Informationen zu Abfahrtszeiten, Häufigkeiten des Fahrten usw. erschweren; 
integrierbar an regionale und überregionale Verkehrsanbieter. Auf die Sicherung von Mobilitätsketten weisen insbesondere Klaus Düwal und Sabine Mertel in der Auswertung Ihres Projekts des Informations- und Leitsystem für Menschen mit geistiger Behinderung am Bahnhof Lüneburg' hin (DÜWAL/MERTEL 2003, 111) hin.

\section{Individuelle Anforderungen an ein Informationssystem zeichnen sich aus durch einen niedrigen Komplexitätsgrad}

- Wenn ich wissen will, wo ein Bus abfährt, muss ich nicht wissen, wie sich der Busbahnhof kartografisch abbilden lässt, d.h. ich muss mich nicht notwendig auf einem Plan orientieren, wenn ich genügend klare Hinweise im realen Kontext erhalte.

- Wenn ich wissen will, wann der nächste Bus abfährt brauche ich nicht den ganzen Fahrplan zu kennen, d.h. Informationen über Abfahrtszeiten müssen aktuell und damit dynamisch sein.

- Ein Netzplan enthält viele Informationen, die ich für meine bestimmte (eingeschränkte) Absicht nicht benötige.

- Wenn ich bereits über genaue Vorstellungen verfüge, möchte ich eine genaue Information.

Ein niedriger individueller Komplexitätsgrad lässt sich durch Reduktion des an sich notwendig komplizierten Netzplans erreichen (Abbildung 3). Das Verständnis des gesamten Liniensystems bleibt erhalten, wird jedoch auf einen Linienstern reduziert. Dieser enthält die wichtigsten Informationen, die Fahrgäste über das Liniennetz benötigen: Nummer, Farbe und Symbol der Linien. Ein individuelles Zielführungssystem für Busnutzer muss jedoch auch stets rekursiv bleiben und so den Zugang zu einer komplexeren Übersicht zulassen (STEIERWALD 2005, 711). 


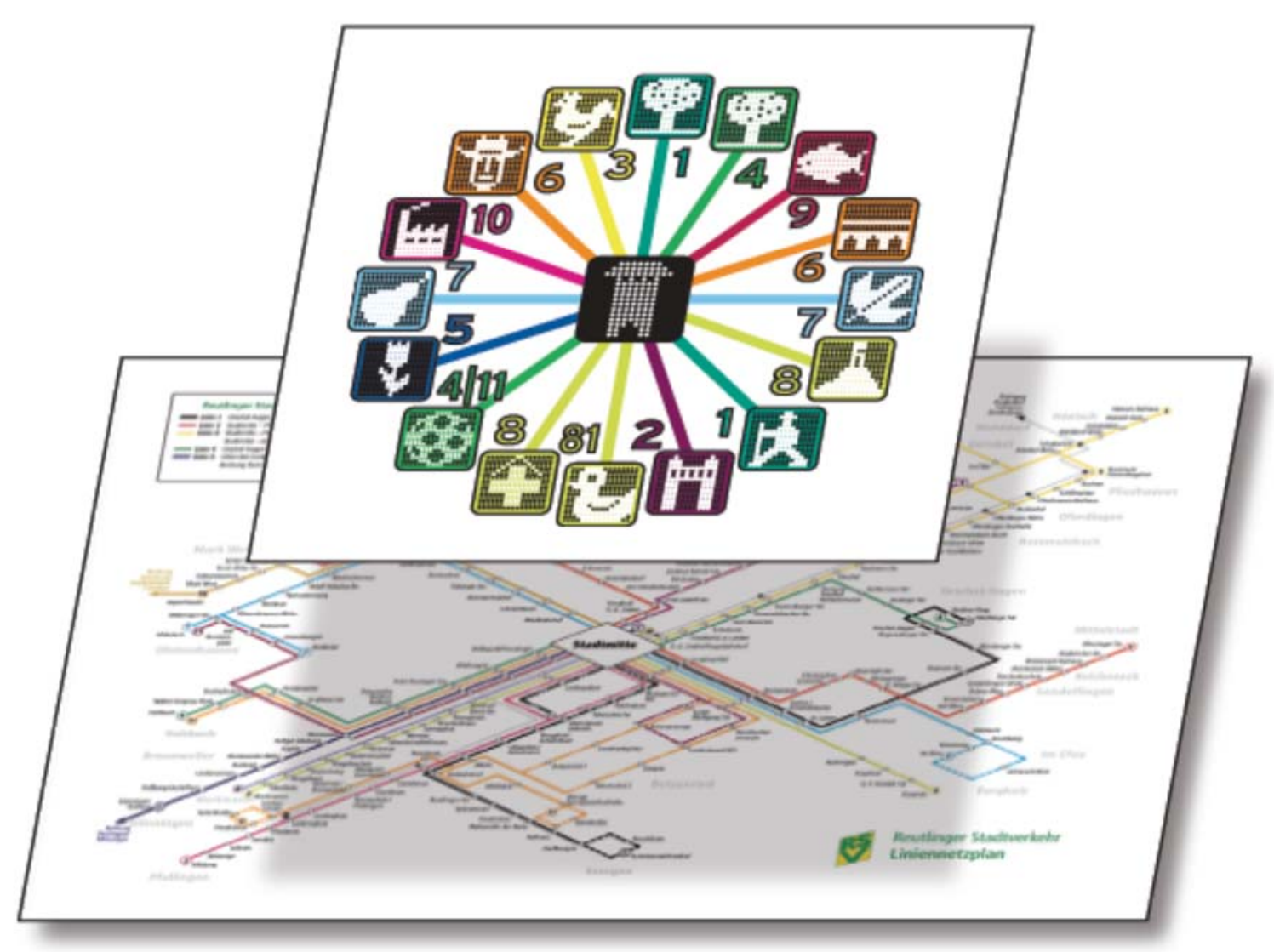

Abbildung 1: Reduktion des komplexen Planes auf Linienstern

\section{Inklusive Anforderungen an ein Informationssystem}

Die Implementierung von Orientierungssystemen für spezifische Zielgruppen in öffentlichen Transportmitteln ist mit einem inklusiven Verständnis von Barrierefreiheit nicht vereinbar. Dies haben bereits die Verantwortlichen des ELLIS-Projekts Lüneburg selbstkritisch eingeräumt. Dazu schreiben sie in ihrem Forschungsbericht: „Das Leitsystem auf dem Lüneburger Bahnhof wird wahrgenommen und verstanden. Wenn auch real kaum jemand der Bahnhofsbenutzer/-innen das Leitsystem benutzt hat, wird doch klar gesehen, wofür es gedacht ist und sein Nutzen wird für eine entsprechende Zielgruppe Orientierung suchender Personen bejaht"

(DÜWAL/MERTEL 2003, 81). Anders als in Lüneburg wurde in Reutlingen aus dem zielgruppenspezifischen Vorhaben „Mobilitätserweiterung für Menschen mit Behinderung" schließlich eine Verbesserung für alle Menschen die mit dem Bus fahren. Menschen mit geistiger Behinderung sind dabei nicht nur „Experten in eigener Sache" (DÜWAL/MERTEL 2003, 77) deren spezifische Bedürfnisse abgefragt und berücksichtigt werden sollen. Vielmehr werden sie als Experten einer gemeinsamen Sache anerkannt, weil sie - wie niemand sonst - das Sensorium und die Aufmerksamkeit für allgemeine Grundsätze der Gestaltung einbringen (vgl. LEIDNER 2007, 401) und sich in der Zusammenarbeit auch so erfahren. Jörg Tröster, ein Mitglied der Arbeitsgruppe Selbstbestimmung formuliert das so: „Das ist 
ja nicht nur für uns, sondern auch für alte Menschen, für Kinder. Und für solche, die sich in Reutlingen nicht auskennen. Die hier einen Besuch machen." 


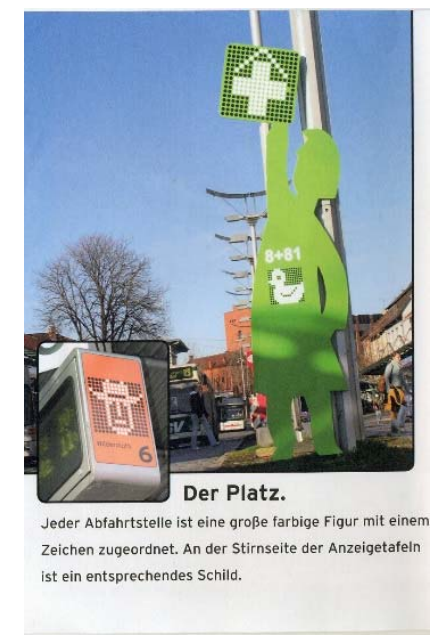

Abbildung 2: Signaturen der Haltestellen

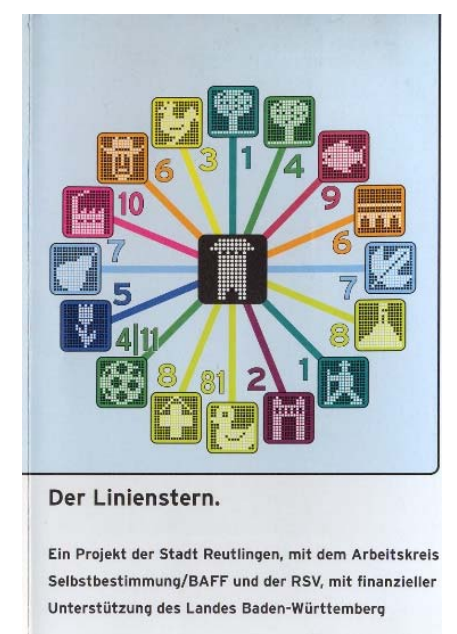

Abbildung 3: Linienstern als Grundmodell

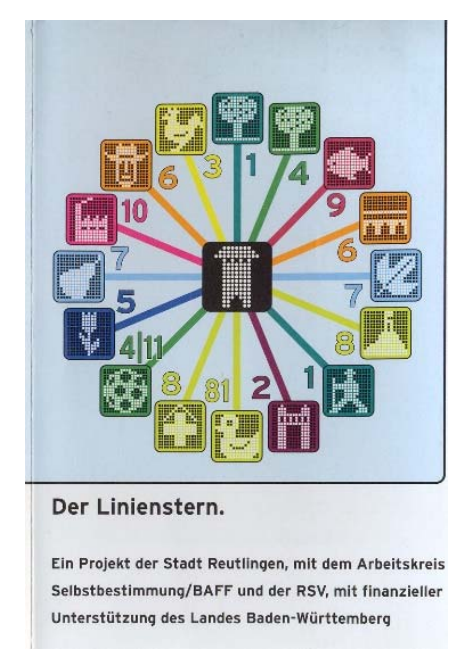

Der Zentrale Omnibusbahnhofes stellt als verkehrliche Schnittstelle (Zielhalt und Umstieg) besonders hohe Anforderungen an Barrierefreiheit, Gestaltqualität. So sorgen hier raumbildende Elemente für schnelle Orientierung der Anordnung, Zugänglichkeit, Sichtbarkeit der Haltepositionen der Busse (vgl. STEIERWALD 2005, 549).

Der Netzplan des Reutlinger

Nahverkehrs ist sternförmig. Alle Linien treffen sich am zentralen Omnibusbahnhof Stadtmitte. Alle Linien haben ein Zeichen, eine Nummer und eine Farbe. Auch die Haltestelle Stadtmitte hat ein Zeichen, nämlich das Tübinger Tor. Die Farben entsprechen den Farben im NALDOPlan der Landkreise Reutlingen, Tübingen und Zollernalb. 


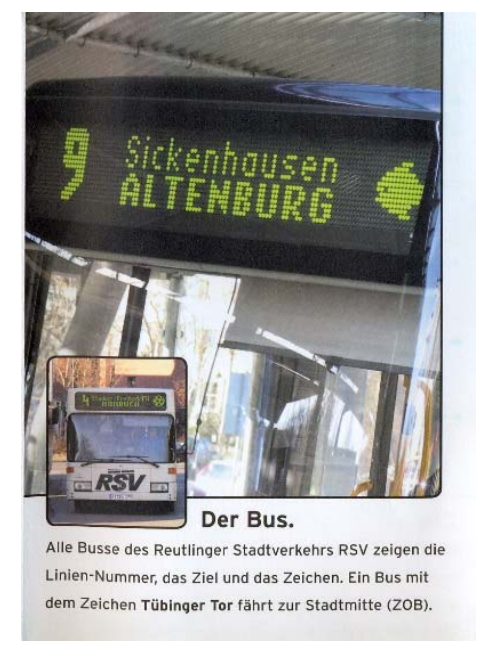

Dynamische Fahrgastinformationen über aktuelle Abfahrt von Bussen an Haltestellen sowie Anzeigen an den Bussen mit Liniennummer, Endhalt und Bildzeichen (STEIERWALD 2005, 711).

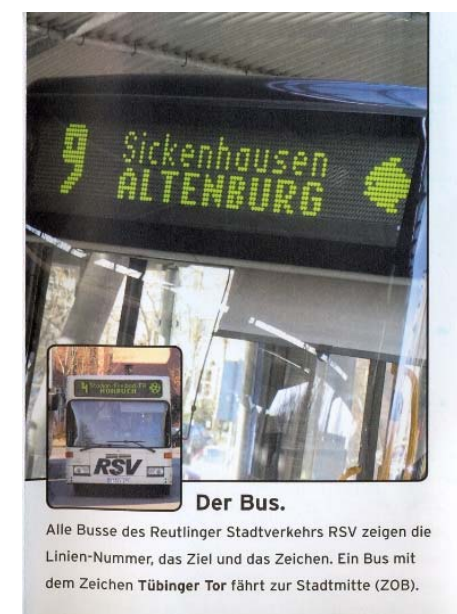

Abbildung 4: Gestaltung der

Displays an Bus und

Haltestelle

Für alle Fahrgäste haben die Mitglieder vom Arbeitskreis Selbstbestimmung eine Einführung und Erklärung des neuen Orientierungssystems formuliert. 


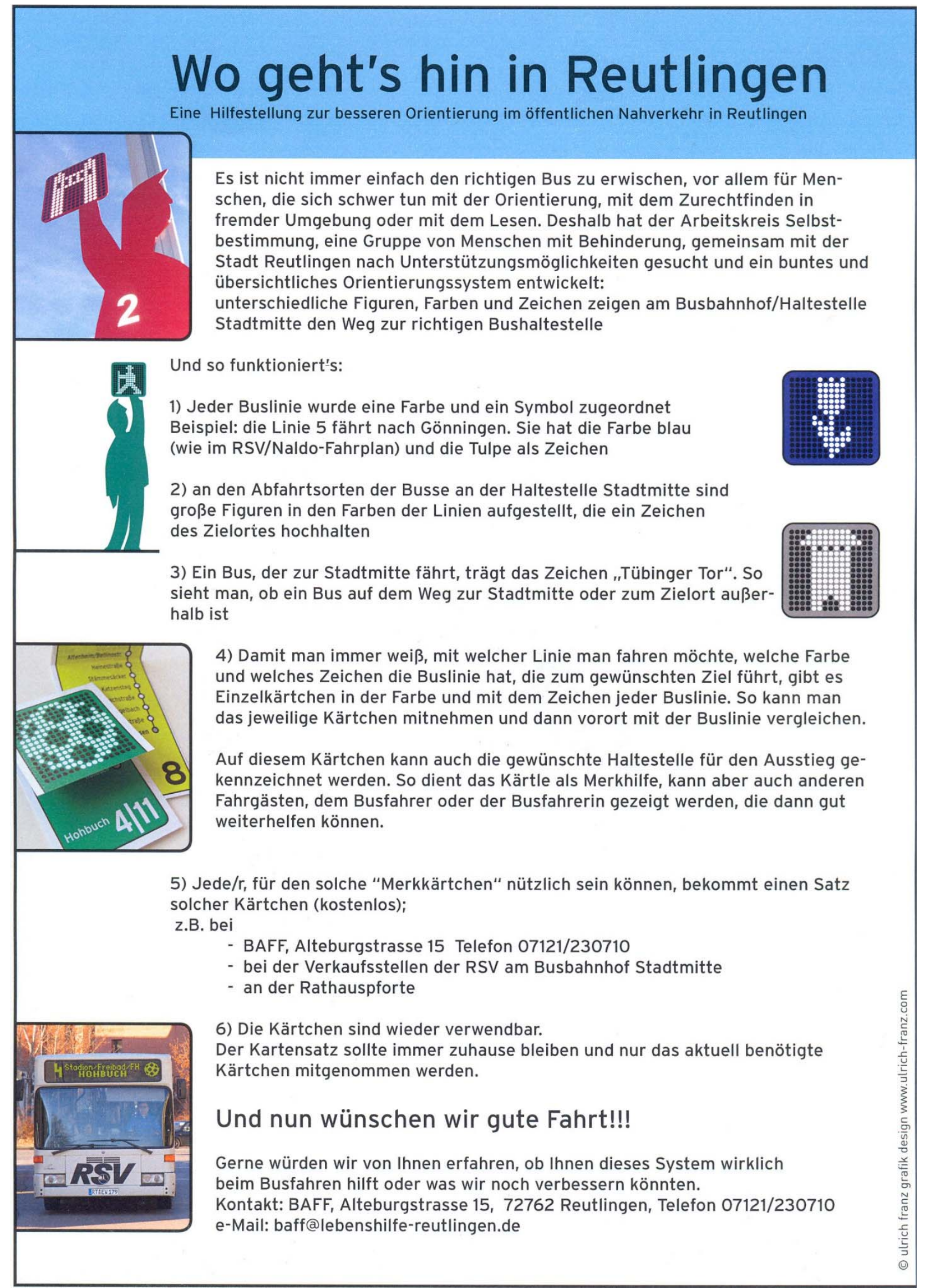

Abbildung 5: Informationsblatt Arbeitskreis Selbstbestimmung 


\section{Evaluation}

Im Abstand von sechs Monaten nach der Einführung wurde im Rahmen einer Befragung die Wahrnehmung dieser Veränderungen und damit der Nutzen einzelner Maßnahmen für die Fahrgäste erhoben und dokumentiert

(GUNZENHAUSER/BAYER 2008). Neben individuellem Training einzelner Menschen mit Lernbeeinträchtigungen im Jugend und Erwachsenenalter, der Ausarbeitung und Erprobung didaktischer Handreichungen für Grund-, Förderschulen und die Schule für Geistigbehinderte in Reutlingen auf dem Hintergrund neuer Bildungspläne ist die Evaluation des Systems selbst die dritte Perspektive der Weiterarbeit am Reutlinger Mobilitätskonzepts (Abbildung 8).

\begin{tabular}{|c|c|c|}
\hline $\begin{array}{l}\text { Nutzungsbezogene } \\
\text { Evaluation }\end{array}$ & individuelles Mobilitätstraining & $\begin{array}{l}\text { didaktische } \\
\text { Handreichungen für } \\
\text { Reutlinger Schulen } \\
\text { (Grund-, Förderschulen } \\
\text { sowie Schule für } \\
\text { Geistigbehinderte) }\end{array}$ \\
\hline $\begin{array}{l}\text { Schließlich geht es in } \\
\text { der dritten Perspektive } \\
\text { um die Erhebung des } \\
\text { Anwendungsnutzens } \\
\text { des implementierten } \\
\text { Orientierungssystems. } \\
\text { Aus gutem Grund wird } \\
\text { dabei als Zugriff nicht } \\
\text { ein Vorher-Nachher- } \\
\text { Verfahren gewählt. } \\
\text { Vergleichserhebungen } \\
\text { sind nachlaufend, } \\
\text { haben } \\
\text { Bestätigungscharakter } \\
\text { und sind wenig } \\
\text { ergiebig. Denn aus } \\
\text { Vergleichserhebungen } \\
\text { lässt sich nur bedingt } \\
\text { eine prospektive Sicht } \\
\text { entfalten. Wir haben } \\
\text { uns deshalb dazu } \\
\text { entschlossen, nach } \\
\text { der Implementierung } \\
\text { des } \\
\text { Orientierungssystems, } \\
\text { die Nutzung einzelner } \\
\text { Elemente des } \\
\text { Orientierungssystems } \\
\text { zu untersuchen. }\end{array}$ & $\begin{array}{l}\text { Die durch individuelles } \\
\text { Training gewonnenen } \\
\text { Erfahrungen geben } \\
\text { Aufschluss über subjektive } \\
\text { Karten, raum- und } \\
\text { Wahrnehmungsorientierungen } \\
\text { von Menschen mit } \\
\text { Lernbeeinträchtigungen und } \\
\text { geben wichtige Hinweise für } \\
\text { die selbständige und } \\
\text { selbstbestimmte Nutzung der } \\
\text { Stadtbusse. Gerade für } \\
\text { Eltern, Assistenten und Lehrer } \\
\text { ist es wichtig, hier } \\
\text { Hilfestellung im Aufbau } \\
\text { verlässlicher und sicherer } \\
\text { Verhaltensweisen zu } \\
\text { bekommen. }\end{array}$ & $\begin{array}{l}\text { Die Bildungspläne der } \\
\text { Grund- und Förderschule } \\
\text { sowie der Schule für } \\
\text { Geistigbehinderte } \\
\text { betrachten Mobilität als } \\
\text { Leitkompetenz für } \\
\text { selbständige } \\
\text { Lebensführung und } \\
\text { Teilhabe. Die Erstellung } \\
\text { und Erprobung } \\
\text { standortbezogener } \\
\text { didaktischer } \\
\text { Handreichungen für die } \\
\text { Reutlinger Schulen stellt } \\
\text { die zweite Perspektive } \\
\text { dar. Dies ist nicht nur } \\
\text { hilfreich für den Einbezug } \\
\text { außerschulischer } \\
\text { Lernorte (wie z.B. Listhof, } \\
\text { Museen und } \\
\text { Stadtbibliothek), Praktika } \\
\text { und } \\
\text { Betriebserkundungen. Die } \\
\text { selbständige Bewältigung } \\
\text { des Schulwegs mit } \\
\text { öffentlichen } \\
\text { Verkehrsmitteln statt } \\
\text { individueller Bring- und } \\
\text { Holdienste durch Eltern } \\
\text { schafft ökologisch } \\
\text { verantwortliches } \\
\text { Mobilitätsverhalten und ist } \\
\text { eine Chance, diese in } \\
\text { einer sehr }\end{array}$ \\
\hline
\end{tabular}




\begin{tabular}{|l|l|}
\hline & Verhaltensprägenden \\
& Lebensphase als \\
& künftigen Kundenstamm \\
& zu gewinnen (vgl. \\
& KOHLER 2002, 118). \\
\hline
\end{tabular}

\section{Kasten 3}

Diese drei unterschiedlichen Zugänge soll durch sog. Multiple Triangulation (DENZIN 1989, KELLE/ERZBERGER 1999, FLICK 2004) eine zukünftige kumulative Validierung ermöglichen. Quantitative und qualitative Elemente können demnach konvergieren/divergieren, d.h. tendenziell übereinstimmen oder sich gegenseitig interpretieren oder sich widersprechen. Wichtige Erfahrungen konnten zwischenzeitlich in der Durchführung und Dokumentation von individuellem Mobilitätstraining gewonnen werden (DORSCH 2007, MÜHLECK 2006, HEINZMANN 2007, SACHS 2008). Dabei überrascht es kaum, dass die Nachhaltigkeit solcher Angebote wesentlich durch das soziale Umfeld, also durch Angehörige und Schule bzw. Betrieb gesichert wird (STÖPPLER 2002, FISCHER 2005, SPITTA 2005, WARWITZ 2005). Bei der Suche nach Arbeits- und Praktikumsstellen für Menschen mit Lernbeeinträchtigungen erweist sich die selbständige Nutzung des ÖPNV die Schlüsselkompetenz.

Welche Menschen nutzen die Reutlinger Stadtbusse? Lassen sich bestimmte Fahrgastgruppen bezüglich der Nutzung vorhandener Orientierungshilfen unterscheiden? Hierzu wurde als angemessenes Instrument die Befragung der Fahrgäste gewählt (STEIERWALD 2005, 128)².

Die Linienkärtchen finden $86 \%$ der Befragten verständlich; $14 \%$ können damit nichts anfangen. $83 \%$ der Fahrgäste orientieren sich an der Liniennummer, $36 \%$ orientieren sich am Endhalt, $14 \%$ orientieren sich am Bild. Für 82\% der Fahrgäste sind die Linienkärtchen Kärtchen verständlich. 47\% können diese Kärtchen für sich oder für einen Angehörigen gebrauchen. Den ausgehängten Fahrplan nutzen 56 \% der Fahrgäste. Für 20\% der Fahrgäste hat sich in der vergangenen Zeit etwas verbessert; $80 \%$ verneinen dies. Anregungen haben $62 \%$ der Fahrgäste $38 \%$ haben keine Anregungen.

Es gibt klar erkennbare Gruppierungen von Fahrgäste mit unterschiedlichen Nutzungsprofilen und Erwartungen; (gesichert durch zweiseitig korrelierende Variablen) bei Geschlecht/Nummernorientierung, Fahrplan/Strecke,

\footnotetext{
${ }^{2}$ Am 14.12.07 sowie am 11.01.08 wurden am ZOB Fahrgastbefragungen durchgeführt. Die beiden Samples (bereinigt n'=99; n"=78) ergeben bezüglich Altersdurchschnitt und Verteilung, Geschlecht, Fahrtanlass, Häufigkeit der Bus-Nutzung keine signifikanten Unterschiede; beide Samples wurden deshalb gemeinsam ausgewertet $(n=177)$. Bei einer durchschnittlichen Anzahl von 50 Tsd.

Beförderungen täglich durch die RSV ist für den Zeitraum Freitag 9-11 Uhr eine geschätzte Anzahl von Nutzern am ZOB von 1500 Personen realistisch (Angaben der Geschäftsleitung RSV und Tiefbauamt Reutlingen); die Anzahl der befragten Personen im og. Zeitraum entspricht demnach ca. $10 \%$ der Nutzer. Die stichwortartige Zusammenfassung dieser Untersuchung (Gunzenhauser/Bayer 2008) soll die nächstens veröffentlichte ausführliche Dokumentation und Auswertung nicht ersetzten. Der Fragebogen sowie die erhobenen Daten der Untersuchung sind als Download „Fahrgastbefragung ZOB RT 2008“ eingestellt http://opus.bsz-bw.de/hsrt/
} 
Häufigkeit/Nutzungszweck, Orientierung Endhalt/Liniennummer/Bild im Display, Nutzungszweck und -häufigkeit/Altersgruppen, Fahrplanlesen/Altersgruppen. Es ließen sich Nutzergruppen identifizieren, die sich gleichwohl nicht durch Alter, Geschlecht, Nutzungshäufigkeit kennzeichnen, sondern eher durch spezifische Wahrnehmungs- und Orientierungsmuster. Die von uns erhobenen Nutzerdaten lassen daher eher andere Individualverhaltensmodelle erkennen als die von Steierwald festgestellten ÖPNV-Personengruppenmodelle (Mobilitätswerte nach Erwerbstätigkeit, Geschlecht, Alter, Ausbildung; vgl. STEIERWALD 2005, 711).

\section{Barrierefreiheit für Menschen mit geistiger Behinderung als gemeinsame Aufgabe}

Neben dem Düsseldorfer Forschungsprojekt ,Barrierefreier Schülerverkehr' von Prof. Dr. Reinhilde Stöppler arbeitet gegenwärtig Prof. Dr. Erhard Fischer an der Universität Würzburg an Fragen zur Räumliche Orientierung und Mobilität bei jungen Erwachsenen mit geistiger Behinderung als Voraussetzung zur Teilhabe am gesellschaftlichen Leben. Die hohe Aufmerksamkeit einer barrierefreien Nutzung von Gebäuden, Verkehrs- und Kommunikationsmitteln und öffentlichen Einrichtungen für Menschen mit Sinnesschädigungen und motorischen Einschränkungen gilt noch nicht in gleicher Weise für Menschen mit geistiger Behinderung (FÖHL 2007, 132). Die Arbeit am Reutlinger Orientierungssystem hat gezeigt, dass sich die diesem Sachverhalt zugrunde liegenden Schwierigkeiten und Herausforderungen nur gemeinsam mit lernbeeinträchtigten Menschen formuliert und bearbeiten lassen. Ihre Expertise kommt allen Fahrgästen zugute. Solche Kooperation in Planung und Implementierung stellt sich weder von selbst ein noch ist sie auf Dauer gestellt. Hier liegt eine wesentliche Entwicklungsaufgabe sonderpädagogischer Aufmerksamkeit der Hochschule als Partner einer forschenden Lehre, die sich auf lokaler Ebene einmischt. Man sagt: „Wo ein Wille ist ist auch ein Weg.“ Liegt in der Umkehrung dieser Binsenweisheit nicht ebensoviel Plausibilität: ist das Nutzenkönnen von Wegen nicht Voraussetzung für die Konkretisierung dessen was man den eigenen Willen (oder zeitgemäßer Selbstbestimmung) nennt?

\section{Literatur}

\begin{tabular}{|l|l|}
\hline $\begin{array}{l}\text { Bayer, Alexander; } \\
\text { Gunzenhauser, Hans- } \\
\text { Martin (2008) }\end{array}$ & $\begin{array}{l}\text { Auswertung der Nutzerbefragung zum Reutlinger } \\
\text { Orientierungssystem. Unveröffentlichte Studienarbeit } \\
\text { Fakultät für Sonderpädagogik Reutlingen. }\end{array}$ \\
\hline $\begin{array}{l}\text { Birkenhauer, Josef } \\
\text { (2004) }\end{array}$ & $\begin{array}{l}\text { Wahrnehmung von Raum und Landschaft bei Kindern und } \\
\text { Jugendlichen. Praxis Geographie 34 (2004)12, 58-60. }\end{array}$ \\
\hline Castner, Henry (1995) & $\begin{array}{l}\text { Orienting ourselves in space implications for the school } \\
\text { curriculum. Toronto, Ontario : Univ. of Toronto Press, 1996 } \\
\text { (= Cartographica : Monograph ; 46. Cartographica ; 32,2). }\end{array}$ \\
\hline Dorsch, Carina (2007) & $\begin{array}{l}\text { Mobilitätserziehung. Unveröffentlichte Studienarbeit Fakultät } \\
\text { für Sonderpädagogik Reutlingen. }\end{array}$ \\
\hline $\begin{array}{l}\text { Downs, Roger M.; Stea, } \\
\text { David (1985) }\end{array}$ & $\begin{array}{l}\text { Kognitive Karten und Verhalten im Raum - Verfahren und } \\
\text { Resultate der kognitiven Kartographie. In: Schweizer, Harro } \\
\text { (Hrsg.) (1985): Sprache und Raum. Stuttgart: Metzler, 18 - } \\
\text { 43. }\end{array}$ \\
\hline
\end{tabular}




\begin{tabular}{|c|c|}
\hline $\begin{array}{l}\text { Düwal, Klaus; Mertel, } \\
\text { Sabine, (2003) }\end{array}$ & $\begin{array}{l}\text { ELLIS-Forschungsbericht Reduzierung von } \\
\text { Mobilitätsbarrieren. Das Pilotprojekt, Informations- und } \\
\text { Leitsystem für Menschen mit geistiger Behinderung am } \\
\text { Bahnhof Lüneburg' } \\
\text { www.hs-zigr.de/ smertel/docs/leitsystem.pdf } \\
\text { (entnommen 05.06.2007). }\end{array}$ \\
\hline Fischer, Erhard (2005) & $\begin{array}{l}\text { Vorhaben und Unterrichtseinheiten: Lehren und Lernen im } \\
\text { Förderschwerpunkt geistige Entwicklung. Dortmund: } \\
\text { Borgmann. }\end{array}$ \\
\hline Flick (2004) & Triangulation. Wiesbaden: Verlag für Sozialwissenschaften. \\
\hline Föhl, Patrick (Hg) (2007) & $\begin{array}{l}\text { Interne Kommunikation für barrierefreie Maßnahmen. In: } \\
\text { ders. (Hg) Das barrierefreie Museum: Theorie und Praxis } \\
\text { einer besseren Zugänglichkeit ; ein Handbuch des } \\
\text { Landschaftsverbands Rheinland zur Museumsberatung. } \\
\text { Bielefeld : Transcript, 131-148 }\end{array}$ \\
\hline $\begin{array}{l}\text { Gramann, Helmut; Görs, } \\
\text { Alexander (2006) }\end{array}$ & $\begin{array}{l}\text { Seniorenspezifischer Tourismus: eine Untersuchung zu den } \\
\text { Ansprüchen älterer Reisender an den Tourismus und zu den } \\
\text { Möglichkeiten der Urlaubsdestinationen dieser } \\
\text { Anspruchshaltung gerecht zu werden. Marburg: Tectum. }\end{array}$ \\
\hline Hard, Gerhard (2003) & $\begin{array}{l}\text { Spuren und Spurenleser. In: ders. Dimensionen } \\
\text { geographischen Denkens. Aufsätze zur Theorie der } \\
\text { Geographie. Göttingen: V\&R Unipress 2003, 165-187 ( = } \\
\text { Osnabrücker Studien zur Geografie Band 23). }\end{array}$ \\
\hline Hasse, Jürgen (2007) & $\begin{array}{l}\text { Übersehene Räume. Zur Kulturgeschichte und } \\
\text { Heterotopologie des Parkhauses. Bielefeld: Transcript }\end{array}$ \\
\hline $\begin{array}{l}\text { Heintzmann, Daniel } \\
\text { (2007) }\end{array}$ & $\begin{array}{l}\text { Das Orientierungssystem der Stadt Reutlingen für den } \\
\text { öffentlichen Nahverkehr: Mobilitätserweiterung für/von } \\
\text { Menschen mit geistiger Behinderung. Wissenschaftliche } \\
\text { Hausarbeit Staatsexamen; Fakultät für Sonderpädagogik } \\
\text { Reutlingen. }\end{array}$ \\
\hline $\begin{array}{l}\text { Herry, M. \& Sammer, G. } \\
\text { (1998) }\end{array}$ & $\begin{array}{l}\text { Mobilitätserhebung österreichischer Haushalte - } \\
\text { Forschungsarbeiten aus dem Verkehrswesen. } \\
\text { Bundesministerium für Wissenschaft und } \\
\text { Verkehr, Wien. }\end{array}$ \\
\hline $\begin{array}{l}\text { Hüttenmoser, Marco } \\
\text { (2005) }\end{array}$ & $\begin{array}{l}\text { Ich gehe also bin ich. Schulweg selbständig und sicher } \\
\text { erleben. Kinder von Balzers Liechtenstein zeichnen ihren } \\
\text { Schulweg im Rahmen des ViaNova Interreg III Projekts } \\
\text { Alpine Space der EU. } \\
\text { http://www.kindundumwelt.ch/de/kindundverkehr.htm } \\
\text { entnommen 10.05.08 }\end{array}$ \\
\hline $\begin{array}{l}\text { Jansen-Osmann, Petra; } \\
\text { Wiedenbauer, Gunnar } \\
\text { (2007) }\end{array}$ & $\begin{array}{l}\text { Wayfinding behaviour and spatial knowledge of adults and } \\
\text { children in a virtual environment. Swiss yournal of } \\
\text { psychology } 66 . \mathrm{Jg}(2007) 1,41-50 \text {. }\end{array}$ \\
\hline $\begin{array}{l}\text { Janzen, Gabriele; } \\
\text { Hawlik, Michaela (2005) }\end{array}$ & $\begin{array}{l}\text { Orientierung im Raum. Befunde zu Entscheidungspunkten. } \\
\text { Zeitschrift für Psychologie } 213 \text { (2005) (4) 179-186. }\end{array}$ \\
\hline $\begin{array}{l}\text { Kelle, Udo; Erzberger, } \\
\text { Christian (1999) }\end{array}$ & $\begin{array}{l}\text { Integration qualitativer und quantitativer Methoden : } \\
\text { Methodologische Modelle und ihre Bedeutung für die } \\
\text { Forschungspraxis. Kölner Zeitschrift für Soziologie und } \\
\text { Sozialpsychologie. Opladen : Westdeutscher Verlag. 51. Jg. } \\
\text { (1999), S. 509-531. }\end{array}$ \\
\hline
\end{tabular}




\begin{tabular}{|c|c|}
\hline $\begin{array}{l}\text { Klippel; Alexander; } \\
\text { Richter, Kai-Florian } \\
\text { (2002) }\end{array}$ & $\begin{array}{l}\text { You-Are-Here Maps: Wayfinding Support as Location Based } \\
\text { System. In J. Moltgen, A. Wytzisk (Eds.), Gl-Technologien für } \\
\text { Verkehr und Logistik. Beiträge zu den Münsteraner GI Tagen, } \\
\text { Vol. 13, IfGI Prints. } \\
\text { http://www.cosy.informatik.uni- } \\
\text { bremen.de/staff/pub.html?user=klippel } \\
\text { (entnommen 18.03.2008) }\end{array}$ \\
\hline Kohler, Sandra (2002) & $\begin{array}{l}\text { Analyse des Mobilitätsverhaltens von Kindern und Eltern als } \\
\text { Basis einer institutionalisierten Mobilitätserziehung. } \\
\text { Technische Universität: München, Dissertation } 2002 \text {. } \\
\text { http://tumb1.biblio.tu- } \\
\text { muenchen.de/publ/diss/ww/2002/kohler.pdf } \\
\text { (entnommen 20.05.2008) }\end{array}$ \\
\hline Leidner, Rüdiger (2007) & $\begin{array}{l}\text { Design für alle. In: Föhl, Patrick }(\mathrm{Hg}) \text { Das barrierefreie } \\
\text { Museum: Theorie und Praxis einer besseren Zugänglichkeit. } \\
\text { Bielefeld: Transcript, 398-408 }\end{array}$ \\
\hline $\begin{array}{l}\text { Mallot, Hanspeter } \\
\text { (2006) }\end{array}$ & $\begin{array}{l}\text { Raumorientierung und kognitive Karten. In: Karnath, Hans- } \\
\text { Otto; Thier, Peter (Hg) Neuropsychologie. Berlin-Heidelberg: } \\
\text { Springer }{ }^{2} 2006,153-159 .\end{array}$ \\
\hline Montello, Dan & $\begin{array}{l}\text { Kartenverstehen in der Kognitionspsychologie. Zeitschrift für } \\
\text { Semiotik. } 20 \text { (1998), S. } 91-103 .\end{array}$ \\
\hline Mühleck, Sophia (2007) & $\begin{array}{l}\text { Mobilitätserweiterung im ÖPNV Reutlingen. Unveröffentlichte } \\
\text { Studienarbeit Fakultät für Sonderpädagogik Reutlingen. }\end{array}$ \\
\hline Sachs, Wiebke & $\begin{array}{l}\text { Mobilitätstraining mit dem neuen Orientierungssystem. } \\
\text { Hausarbeit Staatsexamen; Fakultät für Sonderpädagogik } \\
\text { Reutlingen. }\end{array}$ \\
\hline $\begin{array}{l}\text { Schuhmann-Hengstler, } \\
\text { Ruth (2006) }\end{array}$ & $\begin{array}{l}\text { Räumliche Kognition. In: Schneider, Wolfgang }(\mathrm{Hg}) \\
\text { Enzyklopädie der Psychologie. Göttingen: Hogrefe. }\end{array}$ \\
\hline Spitta, Philipp (2005) & $\begin{array}{l}\text { Praxisbuch Mobilitätserziehung : Unterrichtsideen, Projekte } \\
\text { und Material für die Grundschule. Baltmannsweiler : } \\
\text { Schneider Verl. Hohengehren. }\end{array}$ \\
\hline $\begin{array}{l}\text { Staatsinstitut für } \\
\text { Schulqualität und } \\
\text { Bildungsforschung } \\
\text { (2006) }\end{array}$ & $\begin{array}{l}\text { Perspektiven der Werkstufe. Auswertung einer } \\
\text { Datenerhebung über die Werkstufe des Förderzentrums } \\
\text { Förderschwerpunkt Geistige Entwicklung. Autor: Bruno } \\
\text { Schor; München. }\end{array}$ \\
\hline $\begin{array}{l}\text { Steierwald Gerd (Hg.) } \\
(2005)\end{array}$ & $\begin{array}{l}\text { Stadtverkehrsplanung : Grundlagen, Methoden, Ziele } \\
\text { Berlin/Heidelberg: Springer. }\end{array}$ \\
\hline $\begin{array}{l}\text { Stöppler, Reinhilde } \\
\text { (2002) }\end{array}$ & $\begin{array}{l}\text { Mobilitäts- und Verkehrserziehung bei Menschen mit } \\
\text { geistiger Behinderung. Bad Heilbrunn: Klinkhardt. }\end{array}$ \\
\hline Tully, Claus (Hg) (1999) & $\begin{array}{l}\text { Erziehung zur Mobilität. Jugendliche in der automobilen } \\
\text { Gesellschaft; Frankfurt [u.a.]: Campus. }\end{array}$ \\
\hline $\begin{array}{l}\text { Warwitz, Siegbert } \\
(2005)\end{array}$ & $\begin{array}{l}\text { Verkehrserziehung vom Kinde aus : Wahrnehmen - Spielen - } \\
\text { Denken - Handeln Baltmannsweiler : Schneider, } \\
\text { Hohengehren, 5. Auflage. }\end{array}$ \\
\hline
\end{tabular}

\section{Grafiken:}

\begin{tabular}{|l|l|}
\hline Kasten 1 & aus der Mängelliste des Arbeitskreises Selbstbestimmung \\
\hline Kasten 2 & $\begin{array}{l}\text { individuelle und systematische Voraussetzungen für die } \\
\text { Gestaltung }\end{array}$ \\
\hline Abbildung 1 & $\begin{array}{l}\text { Reduktion des Liniennetzes auf Linienstern; CUlrich Franz mit } \\
\text { freundlicher Genehmigung }\end{array}$ \\
\hline
\end{tabular}




\begin{tabular}{|l|l|}
\hline $\begin{array}{l}\text { Abbildungen } \\
2-4\end{array}$ & $\begin{array}{l}\text { Gestaltung des Omnibusbahnhofs, der Linienübersicht und der } \\
\text { Displays an Bussen und Haltestellen }\end{array}$ \\
\hline Abbildung 5 & Informationsblatt des Arbeitskreises Selbstbestimmung \\
\hline
\end{tabular}

Das Bildmaterial des Reutlinger Orientierungssystems sowie weitere Informationen sind eingestellt unter https://www.stadtwerke-reutlingen.de/rsv/00 home/00 home01.htm; die „Fahrgastbefragung ZOB RT 2008“ ist als pdf-Dokument eingestellt http://opus.bsz-bw.de/hsrt/ 